\title{
TTR
}

Traduction, terminologie, re?daction

\section{Problèmes de traduction du vernaculaire noir américain : le cas de The Adventures of Huckleberry Finn}

\section{Judith Lavoie}

Volume 7, numéro 2, 2e semestre 1994

Traduire les sociolectes

URI : https://id.erudit.org/iderudit/037183ar

DOI : https://doi.org/10.7202/037183ar

Aller au sommaire du numéro

Éditeur(s)

Association canadienne de traductologie

ISSN

0835-8443 (imprimé)

1708-2188 (numérique)

Découvrir la revue

Citer cet article

Lavoie, J. (1994). Problèmes de traduction du vernaculaire noir américain : le cas de The Adventures of Huckleberry Finn. TTR, 7(2), 115-145.

https://doi.org/10.7202/037183ar

\section{Résumé de l'article}

Problèmes de traduction du vernaculaire noir américain : le cas de The Adventures of Huckleberry Finn - Cet article propose une analyse du rôle dévolu au vernaculaire noir américain (VNA) par Mark Twain dans The Adventures of Huckleberry Finn et du traitement qui en a été fait par deux traducteurs français (Suzanne Nétillard, 1948/1973/1985, et André Bay, 1961/1990). L'auteure démontre que la transcription du VNA par Twain répond à deux « tendances esthético-cognitives divergentes » (Lane-Mercier). La première, " philologique ", où Twain tente, sans vraiment y parvenir en raison de certains effets de clôture, de rendre compte du parler des personnes de race noire dans l'extratexte; la seconde, " artistique ", où il cherche à subvertir, à travers sa représentation du VNA sur le plan scriptural, le discours socio-idéologique propre à sa société. En effet, le VNA assume plusieurs fonctions dans The Adventures of Huckleberry Finn: sur le plan esthétique, il crée, au début du roman, un effet de comique; sur le plan social, il identifie le locuteur à son milieu; et sur le plan idéologique, il exprime la position de l'auteur sur l'esclavage et la ségrégation. Or, la tradition française du bien-écrire étant très présente à l'esprit des traducteurs, ces derniers ont plus ou moins pu recréer graphiquement en français un langage caractérisant la voix noire tel que Twain l'avait fait en anglais. Partant, si le VNA n'est pas représenté formellement, toute l'idéologie sous-jacente à sa présence est du même coup atténuée, si ce n'est complètement perdue.
Tous droits réservés @ C TTR: traduction, terminologie, rédaction — Les auteurs, 1994 ce document est protégé par la loi sur le droit d'auteur. L’utilisation des services d'Érudit (y compris la reproduction) est assujettie à sa politique d'utilisation que vous pouvez consulter en ligne.

https://apropos.erudit.org/fr/usagers/politique-dutilisation/ 


\section{Problèmes de traduction du vernaculaire noir américain: \\ le cas de The Adventures of Huckleberry Finn ${ }^{1}$}

\section{Judith Lavoie}

\section{Introduction}

En 1876, Mark Twain confie à William Dean Howells qu'il a commencé la rédaction d'un roman dont il est relativement fier, mais ajoute qu'il mettra de côté son manuscrit ou le brûlera après l'avoir terminé2. Ce roman, The Adventures of Huckleberry Finn ${ }^{3}$, n'a été ni détruit ni rangé dans un fond de tiroir, mais publié aux États-Unis au début de l'année 1885, sept ans après sa mise en chantier. The Adventures of Huckleberry Finn est une œuvre qui

1. L'auteure remercie Annick Chapdelaine et Jean-Marc Gouanvic de leur aide dans la rédaction de cet article. Elle exprime également sa reconnaissance à Michael Gilson qui a traduit le résumé.

2. Lettre de Twain à Howells citée par Harold Beaver (1987, p. 20) d'après Henry Nash Smith (1960, p. 144).

3. Nous nous sommes servie de l'édition de Sculley Bradley et al. (1977). Les extraits tirés de ce texte seront suivis entre parenthèses des lettres $H F$ et du numéro de la page. 
marquera toute la littérature américaine en raison des nombreux sociolectes qu'elle met en présence, lesquels offrent la possibilité «[...] of using vernacular speech for serious purposes and of transforming the vernacular narrator from a mere persona into a character with human depth» (Smith, 1962, pp. 113-114). Le sociolecte auquel nous nous intéresserons est celui des locuteurs noirs du roman, le vernaculaire noir américain (VNA) ${ }^{4}$. Cet article comporte deux parties: la première est orientée vers l'analyse formelle de l'inscription du VNA dans The Adventures of Huckleberry Finn; la seconde porte plus précisément sur les fonctions qu'assume le VNA dans le texte. Une étude descriptive des choix de traduction de deux traducteurs français sera effectuée dans le développement des deux parties.

Nous avons repéré dix traductions de The Adventures of Huckleberry Finn'. Nous analyserons deux d'entre elles, la traduction de Suzanne Nétillard parue pour la première fois en 1948

4. Nous empruntons cette appellation à William Labov $(1972$, p. 189; 1976 , p. 264, pour la traduction) dont le traducteur, Alain Kihm, a ainsi traduit le syntagme anglais Black English Vernacular (BEV).

5. Voici ces dix traductions accompagnées de la date de leur première parution: les Aventures de Huck Finn, ami de Tom Sawyer, tr. de William L. Hughes (Paris, Hennuyer, 1886); les Aventures de Huck Finn, tr. de Yolande et René Surleau (Paris, Hachette, Bibl. Verte, 1946-48); les Aventures d'Huckleberry Finn, tr. de Suzanne Nétillard (Paris, Éditions Hier et Aujourd'hui, 1948); les Aventures d'Huckleberry Finn, tr. d'André Bay (Paris, Livre club du libraire, 1961); les Aventures de Huckleberry Finn, adapt. d'André-V. Pierre (Paris, Delagrave, «Biblioth. Juventa», 1962); les Aventures de Huck Finn, tr. de P.-F. Caillé (Paris, Hachette, Coll. "Trois en Un», 1965); les Aventures de HuckleberryFinn, tr. de Jean La Gravière (Paris, Éditions G.P., 1979); HuckleberryFinn, tr. de Claire Laury (Paris, Éditions Lito, 1979); Huckleberry Finn, tr. d'Hélène Costes (Neuilly-sur-Seine, Dargaud, 1980); les Aventures de Huckleberry Finn, tr. de Claire Trocmé (Évreux, Diffusion guilde du disque, 1982). 
et réimprimée en 1985 dans la collection «Folio junior» (qui reprenait le texte des Éditions La Farandole de 1973), et celle d'André Bay d'abord publiée en 1961 et reprise en 1990 aux éditions Laffont ${ }^{6}$.

De manière générale, les sociolectes, dont fait partie le VNA, sont surtout présents au sein de la composante dialogale d'une œuvre romanesque. Or, selon Gillian Lane-Mercier, on peut dégager de l'étude des dialogues romanesques,

[deux] tendances, esthético-cognitives divergentes, orientées, d'un côté, vers la volonté «philologique» de connaître le réel discursif, au moyen d'effets de mimésis soigneusement aménagés, de métadiscours visant l'exhaustivité; de l'autre, vers la volonté "artistique» de forger des mondes verbaux possibles, des discourslimites, qui font néanmoins connaître, en creux, les ouvertures paradigmatiques, l'aspect mouvant, créateur de la parole réelle. (Lane-Mercier, 1989, p. 21)

À première vue, le projet esthétique de Twain semble davantage s'apparenter à la première tendance qui vise à créer un effet de réel grâce à l'utilisation de divers procédés de transcription linguistique. Toutefois, il faut se garder de n'attribuer à Twain que des visées de reproduction du réel; son projet esthétique présuppose également le désir de subvertir la réalité, comme la présence du VNA dans The Adventures of Huckleberry Finn en témoigne. Il y a chevauchement,

6. Mark Twain, les Aventures d'Huckleberry Finn, traduit de l'américain par Suzanne Nétillard (Paris, La Farandole, 1973, reprise par Gallimard, Coll. «Folio junior», 1985 pour notre édition), 381 p.; les Aventures d'Huckleberry Finn, l'ami de Tom Sawyer, traduit de l'américain par André Bay (Paris, Robert Laffont, Coll. «Bouquins», 1990 pour notre édition), pp. 177-385. Précisons que nous avons choisi ces textes parce qu'ils étaient facilement accessibles. Il est important de noter que ces deux textes ont été réédités dans deux collections se situant à l'opposé l'une de l'autre, la première, «Folio junior», s'adressant à un public d'adolescents alors que la seconde, «Bouquins», vise un public d'adultes. 
à l'intérieur de cette œuvre, des deux tendances esthético-cognitives définies par Lane-Mercier. C'est d'ailleurs ce qu'a constaté Hans Bungert dans son étude sur l'influence de la tradition humoristique du Sud chez William Faulkner pour qui

Mark Twain [...] obéit au principe du pars pro toto [la partie pour le tout]; au lieu de noter "pains-takingly» tous les détails et nuances du dialecte (comme il l'affirme dans la préface de The Adventures of Huckleberry Finn), il eut recours à de simples marqueurs signalétiques. De l'oralité du texte original naît une langue authentique, compréhensible, littéraire, sans que soit entraînée une diminution de la puissance du réel ${ }^{7}$.

En effet, comme nous le verrons, Twain est plus ou moins parvenu à respecter son projet initial censément réaliste; en fait, il a surtout donné un rôle d'indicateur au VNA, indicateur de sa propre position socio-idéologique. Par l'étude des paroles de Jim, l'esclave en fuite avec Huckleberry, nous tenterons de voir comment les traducteurs ont traité cette dimension de l'œuvre.

\section{Le VNA sur le plan formel}

Avant d'aborder l'étude du VNA sur le plan strictement formel, il est important de faire une distinction entre les intentions déclarées de l'auteur en ce qui touche son utilisation d'un sociolecte et l'inscription véritable de ce sociolecte dans le texte. Le projet avoué de Twain et sa représentation formelle du VNA sont en effet

7. Hans Bungert, William Faulkner und die humoristische Tradition des amerikanischen Südens (Heidelberg, Carl Winter Universitätsverlag, 1971), p. 134: «[...] verfolgt Mark Twain ein Prinzip des pars pro toto; statt "painstakingly" alle Merkmale und Nuancierungen des Dialekts zu verzeichnen (wie er in seiner Vorbemerkung zu Huckleberry Finn behauptet), begnügt er sich mit Signalwerten. Aus dem mundartlichen Rohmaterial wird so eine durchgestaltete, allgemein verständliche, literarische Sprachform, ohne daß dabei eine Minderung der realistischen Wirkung einträte.» [Traduction française de Sophie Boivin.] 
presque à l'opposé l'un de l'autre. Bien que l'auteur nous semble déterminé à reproduire systématiquement certains parlers utilisés autour de lui, entre autres le VNA, sa transcription est partielle; elle est approximative, voire arbitraire. Bien loin de rendre justice au projet de départ, cette transcription est souvent décentrée. David R. Sewell, spécialiste des langages dans l'œuvre de Twain, a aussi constaté le décentrement que subit le projet mimétique de l'auteur:

Despite the apparent empirical pretensions of the «Explanatory» note to Huckleberry Finn, Twain's project was different: he created a literary simulacrum of linguistic diversity that exaggerates and stylizes the heteroglossic interweaving of speech types in real societies. (Sewell, 1987, p. 109)

Nous reviendrons, dans la seconde partie de notre étude, sur les fonctions que peut assumer le VNA sur le plan littéraire puisque, comme le fait remarquer Lane-Mercier:

[La] vraisemblance phonologique des échanges littéraires ne peut être pensée qu'en corrélation avec les propriétés structurales, fonctionnelles et idéologiques intrinsèques à l'œuvre, qui attribuent à tel personnage tel accent, tel tic linguistique, telle structure syntaxique pour des raisons strictement sémiotiques [...]. (1989, p. 169)

Nous verrons, en effet, que le VNA est surtout signe textuel avant d'être imitation du réel, signe du comique, du social et de l'idéologique.

\subsection{Le projet avoué de Twain}

Twain prévient le lecteur dans une note explicative placée en tête de son livre que The Adventures of Huckleberry Finn contient de nombreux sociolectes, dont le «Missouri Negro dialect», et que «[the] shadings have not been done in a hap-hazard fashion, or by guess-work; but pains-takingly, and with the trustworthy guidance and support of personal familiarity with these several forms of 
speech» $(H F, 2)$. Le projet de Twain est explicitement mimétique ${ }^{8}$ lorsqu'il retranscrit les sociolectes. Tel a d'ailleurs été le constat de Janet H. McKay (1982) dans son étude sur la littérature américaine: "Twain's passionate commitment to the effective use of language was the basis of his realistic technique.» (p. 141) Méticuleux, Twain a constamment recherché le bon son pour chaque mot. ${ }^{9}$ La dernière phrase de sa note explicative est instructive: «I make this explanation for the reason that without it many readers would suppose that all these characters were trying to talk alike and not succeeding) $(H F, 2)$. Cette dernière explication permet de mesurer toute l'importance que revêtait pour Twain l'utilisation de parlers vernaculaires. Sewell (1987, p. 101) affirme pour sa part qu'il a ajouté cette note pour ne pas être accusé de mal maîtriser les sociolectes littéraires ${ }^{10}$. Pour nous, le seul fait d'inclure une explication de ce genre témoigne de l'humour de Twain, mais également d'une stratégie visant à orienter la réception de son texte par le lecteur; obligeant ce dernier à porter une attention particulière à cet aspect de l'œuvre, il crée peut-être, par la même occasion, une

8. Voir l'une des notes de ses cahiers: " "Let Jim say putty for 'pretty' \& nuvver for "never" [environ 1879-80] ", cité par Beaver (1987, p. 25) d'après Bernard DeVoto (1942) et Walter Blair (1960).

9. Beaver (1987) signale que Twain a apporté environ mille modifications à son manuscrit avant de l'envoyer à son éditeur, sans compter «[...] such economies as the elimination of italics for emphasis» (p. 29).

10. Sewell considère également que la précaution de Twain faisait indirectement allusion à la grande entreprise du XIX ${ }^{e}$ siècle qui promulguait un enseignement prescriptif de la langue, lequel englobait la façon d'écrire (orthographe et grammaire), mais aussi la façon de converser avec autrui. Autrement dit, il serait question ici, selon Sewell, de comportements paralinguistiques, de maximes conversationnelles, d'actes illocutoires, phénomènes relevant principalement de la pragmatique, aspect auquel s'intéresse beaucoup Sewell (voir pp. 101-109). 
fausse piste de lecture. Le lecteur qui nous intéresse ici est le traducteur, pour qui cette note aurait dû prendre une importance capitale. Comme le fait remarquer Antoine Berman (1984),

[1] traducteur doit «se mettre en analyse», repérer les systèmes de déformation qui menacent sa pratique et opèrent de façon inconsciente au niveau de ses choix linguistiques et littéraires. (p. 19)

Suzanne Nétillard, pour sa part, n'a pas traduit la note explicative de Twain. Cela s'explique peut-être par le public surtout composé de jeunes que vise sa traduction ou par une contrainte émanant de la maison d'édition. Quoi qu'il en soit, ne pas traduire cette note, c'est ne pas attirer l'attention sur une dimension de l'œuvre qui posait bien des difficultés à la traductrice. Nous serions en présence d'une stratégie, non plus de l'auteur, mais de la traductrice qui, pourtant (nous le verrons plus loin), nous semble avoir déployé davantage d'efforts pour reproduire la diversité sociolectale de cette œuvre que son homologue masculin, André Bay. Paradoxalement, ce dernier a traduit la note mais d'une manière tout à fait particulière. Ce qui, dans l'original, apparaissait en style direct, l'auteur s'adressant directement à nous, est inclus, dans la version de Bay, dans une «note sur la traduction». En fait, le traducteur a rapporté en substance une partie des paroles de l'auteur. Bay n'énumère pas tous les sociolectes que Twain prenait la peine de mentionner et il ne dit pas que ces derniers n'ont pas été reproduits «in a hap-hazard fashion or by guess-work; but painstakingly», etc. Il ajoute cependant que:

En employant systématiquement le style parlé, Mark Twain, en autodidacte conscient de ses limites, a opéré une véritable révolution dans la littérature américaine, il lui a permis d'être enfin elle-même et non plus une branche de la littérature anglaise. Dans la présente traduction, nous nous sommes efforcés [sic] de conserver au récit son naturel un peu débraillé, familier, sans aller jusqu'à l'argot et en respectant autant que possible les règles de la grammaire. (Bay, p. 178) 
Nous serions portée à voir là les limites, non pas de Twain, mais de l'institution littéraire française à laquelle Bay appartient. En fait, sa stratégie est de prévenir le lecteur que le projet de Twain posait un problème de taille à la traduction, par conséquent au polysystème littéraire français, d'où son dernier commentaire sur le respect des règles de la grammaire. Comme le souligne Annie Brisset, la traduction «[fait] apparaître les tensions du système et renseigne par là sur les conditions régissant les échanges littéraires et plus généralement les échanges culturels dans un état de société») (Brisset, 1990, p. 317).

\subsection{L'inscription véritable du VNA dans le texte}

Comme nous le notions plus haut, malgré ses intentions prétendument réalistes, Twain n'a pas réussi à retranscrire le VNA aussi fidèlement qu'il le déclarait. Obligé de se plier aux «exigences de lisibilité» (Lane-Mercier, 1989, p. 164) du romanesque, Twain s'est vu contraint de faire des choix; autrement dit, il n'a pas pu marquer tout le discours de Jim de manière systématique. Agnès Whitfield et Gregory Lessard l'ont constaté dans une étude sur l'inscription du joual dans le roman québécois, les romanciers ne reproduisent pas scrupuleusement le sociolecte tel qu'ils l'entendent dans le réel: ils «[...] n'utilisent en fait qu'une sous-classe des caractéristiques linguistiques du parler populaire québécois, qu'il s'agisse de phénomènes phonétiques, lexicaux, syntaxiques ou sémantiques» (Whitfield et Lessard, 1991, p. 145). Nous avons constaté que Twain n'avait lui aussi eu recours qu'à une sous-classe d'éléments scripturaux pour identifier le VNA, il n'aurait donc pas échappé à cet «effet de clôture» dont parle Lane-Mercier (1990):

[...] tout dialogue romanesque ne contient qu'un nombre plus ou moins limité de particularités pragmatico-linguistiques caractéristiques du parler réel représenté, et ce en raison d'un tri tout à fait conscient effectué par l'auteur parmi ces dernières. Soulignons que ce tri peut opérer à de multiples niveaux et, dans tous les cas, donne lieu à des réaménagements divers qui «réduisent» le taux de réalisme des passages dialogués. [...] De plus, les traits pragmatico-linguistiques effectivement retenus par l'auteur ont tendance à se systématiser au sein du texte et, ce faisant, à 
engendrer des réseaux de redondances, si bien que [...] ce sont précisément ces fermetures, entre autres, qui assurent la spécificité des dialogues romanesques. (p. 61, n. 10) ${ }^{11}$

Ce seront ces «réseaux de redondances» qui feront que l'on reconnaîtra le locuteur à ses seules paroles. Il est intéressant de remarquer, comme premier effet de clôture, que certains marqueurs phonétiques, comme l'élision du phonème final [er] que l'on retrouve dans «sir» par exemple, ne s'étend pas à tous les morphèmes qui présentent cette terminaison. Seul «sir» devient dans le parler de Jim, «sah», mais «nigger», «trader», «father», «preacher», «river», «better», sont rapportés tels quels, même s'il eût été réaliste de lire «niggah», «tradah», «fathah», etc. Ces morphèmes semblent quelque peu difficiles à lire; pourtant, Twain fait dire à Jim «heah» pour «here», «dah» pour «there», «whah» pour "where». De la même manière, Twain a parfois éliminé les phonèmes finals $[\mathrm{t}]$ comme dans «wouldn'», «res'», «breakfas'» et [r] comme dans «do'», «sho'», «b'fo'», mais «couldn't» est resté tel quel et «went» n'est pas devenu «wen'», ni «noticed», «notice'»; pas plus que «Lord» ne devient «Lo'», ni «for» ne devient «fo'». C'est donc la répétition de certains marqueurs comme le «d» qui remplace le «th» dans des morphèmes comme «that», «they», «them», «these» qui deviennent «dat», «dey», «dem» et «dese» systématiquement, ou encore la graphie du verbe «was» qui devient «wuz» ou "'uz» dans la plupart des cas, ou la récurrence de certaines graphies comme «nuffn» pour «nothing», «sumfn» pour «something», «awluz» pour «always», «gwyne» pour «going to», «kase» pour «cause» ou encore «bekase» pour «because», qui font que le lecteur reconnaît le locuteur dès qu'il prononce quelques mots. ${ }^{12}$

11. Précisons que nous ne retiendrons que les marqueurs linguistiques, et non pas les marqueurs pragmatiques, comme le fait plus spécifiquement Sewell, tel que nous le mentionnions à la note 10 .

12. Il est important de noter que certaines de ces graphies, bien qu'elles apparaissent plus souvent dans les répliques de Jim ou des autres esclaves, seront visibles également chez d'autres 
Avant d'aborder l'analyse des traductions, précisons que nos observations s'inspirent directement et indirectement de la pensée bermanienne de la traduction, qui prend en compte forme et fond de la traduction, de la «[...] traduction-de-la-lettre, du texte en tant qu'il est lettre» (Berman, 1985, p. 45). C'est pourquoi nous porterons notre attention sur la recréation ou l'effacement d'un «jeu de signifiants» (p. 36) propres à la voix noire de cette œuvre parce qu'ils sont porteurs de sens, de messages, qui, si le parler noir est gommé, sont eux aussi perdus. Or, sur le plan lexical, ni la traduction de Nétillard, ni celle de Bay, n'ont su recréer un réseau de redondances comparable à celui de Twain. La tentative est tout de même présente chez Nétillard où des morphèmes comme "quéque» pour «quelque» (on voit régulièrement «quéque chose» ou «quéque part»), «pasque» pour "parce que», «pis» pour «puis», «neyé» pour «noyé», apparaissent souvent dans le discours de Jim. Bien que ces éléments linguistiques s'apparentent davantage à un parler «paysan» que noir, ils marquent plus spécifiquement la voix du personnage noir à l'intérieur de la traduction. C'est pourquoi nous pouvons admettre que Nétillard a tenté de recréer un ensemble de termes qui caractérisent le locuteur, mais sans qu'il n'atteigne l'envergure de celui de Twain. Nous reviendrons sur les enjeux de la traduction du VNA par un vernaculaire rural.

La traduction de Bay, quant à elle, ne reproduit pas un système de marqueurs sociolectaux que le lecteur pourrait associer à la parole noire, comme c'est le cas en anglais. Elle expose, en fait, une des tendances déformantes signalées par Antoine Berman, «la

personnages. Lee A. Pederson fait remarquer que non seulement les personnages noirs disent «awluz», mais aussi un personnage blanc, appelé le «King»; de même pour l'élision du «r» qui est aussi partagée par un personnage blanc, Boggs, ce représentant de l'«extremest [sic] form of the backwoods South-Western dialect». Nous avons constaté également que ce même Boggs disait "gwyne» pour "going to» $(H F, 115)$. De plus, Pederson (19651966, pp. 1-4) signale que seuls les personnages noirs remplacent le «d» par le «th» et disent «kase» et «bekase» pour «cause» et «because». 
destruction des réseaux langagiers vernaculaires», laquelle consiste en l'effacement de «[la] visée polylingue de la prose [qui] inclut forcément une pluralité d'éléments vernaculaires» (1985, p. 78). Ce gommage presque systématique des sociolectes dans la traduction de Bay relèverait de la tradition française du bien-écrire qui caractérise le polysystème littéraire auquel le traducteur appartient. Ce polysystème impose, à lui seul, des normes du traduire auxquelles le traducteur peut se soumettre sans même s'en apercevoir. Aussi ce dernier aura-t-il recours à un procédé particulier pour que, sans que cela ne soit visible sur le plan de la graphie, le lecteur puisse imaginer le parler truffé de déviations du personnage de Jim. En effet, la toute première réplique de Jim ne porte aucun marqueur pouvant identifier la voix noire, mais la seconde l'est à un degré tout à fait étonnant si on la compare à celles qui suivront. Cette seconde réplique contient à elle seule sept élisions de la lettre «e» en trois lignes: dans la conjonction «que», dans le pronom personnel «je» et dans le pronom démonstratif «ce». Cependant, et c'est ce qu'il faut noter, Bay n'aura presque plus recours à l'élision dans les autres prises de parole de Jim. Il semblerait donc que la stratégie du traducteur soit d'inclure un grand nombre de marqueurs au sein d'une seule réplique, pour compter ensuite sur la collaboration du lecteur qui devra, lorsque Jim reprendra la parole, opérer mentalement les multiples déviations linguistiques qui le caractérisent. Cette façon de traduire démantèle la logique de l'œuvre de départ et, du coup, détruit ses systématismes.

L'apostrophe est un marqueur abondamment utilisé par Twain et qui est consigné dans la littérature écrite américaine autant que française pour indiquer l'élision d'une ou de plusieurs lettres. Il faut toutefois noter que cet indicateur n'est pas réservé aux locuteurs du VNA, mais qu'il s'agit d'un saupoudrage se manifestant à travers l'ensemble de l'œuvre, comme en témoigne cet extrait d'une réplique $d u$ «King»: «[...] and so what's the use o' your bein' sour? It'll only make things oncomfortable. [...] Make the best $o$ ' things the way you find 'em, says I — that's my motto» $(H F, 102)$. Les paroles de Jim sont également truffées de ce type d'élision, et il semble que Nétillard l'ait pris en considération, sa traduction présentant elle aussi des élisions dans les répliques de 
Jim, de l'unité graphique finale [re] surtout. Jim dira souvent «attend'», "pauv'», «rent'ent», «aut'», «entend'», «nèg'», «arb'», mais aussi «possib'», «centup'», «i'», où ce sont les lettres «le» ou «l» qui sont éliminées. Il est évident que Nétillard a réellement tenté d'imiter Twain dans ses tentatives de reproduire le réel linguistique.

Twain a égalementrecours à des marqueurs morphologiques dans sa transcription sociolectale. Ceux que l'on retrouve le plus souvent chez Jim se caractérisent par des conjugaisons déviantes comme «I is», «I creeps», «you is», «dey knows», ou encore «I begin» et «she try» pour «I began» et «she tried», bien que parfois il conjugue selon les règles du bon usage grammatical, comme c'est le cas pour «I hear», «I see», «I stole», «I swum», «I had» $(H F, 39$ et 40 ). Ce genre de déviations morphologiques s'observe chez d'autres personnages, mais les occurrences les plus nombreuses apparaissent chez les locuteurs noirs. Ces déviations qui les identifient sont plus difficiles à traduire en français à cause du poids des normes grammaticales dans la société-cible.

Dans une certaine mesure, la traduction de Nétillard parvient à reproduire ce genre de déviations. À Jim il reviendra de faire en français des erreurs morphologiques du type: «je m'ai sauvé», «je m'ai caché», «je m'ai ruiné». Dans la traduction de Bay, la deuxième prise de parole de Jim est la plus marquée. Les déviations morphologiques et syntaxiques sont, par exemple: «où qu'vous êtes» au lieu de «où est-ce que vous êtes», «faut pas» au lieu de «il ne faut pas», et «j'vas» au lieu de «je vais», «qui c'est» au lieu de «qui est-ce». Toutefois, plus aucune autre réplique ne sera à ce point truffée de marqueurs signalétiques. Jim fera bien parfois une ou deux élisions de la négation «ne», mais sans plus. Au contraire, il se mettra même à utiliser le présent et le passé du subjontif. Fidèle à son programme, Bay (1990) a traduit «en respectant autant que possible les règles de la grammaire ${ }^{13} \gg($ p. 178).

13. Nous reproduisons en annexe un extrait des répliques de Jim en anglais accompagné de ses deux traductions afin de voir la voix française de Jim, non plus fragmentée, mais dans son ensemble. 
Ce qui distingue les choix de traduction de $\mathrm{S}$. Nétillard de ceux d'A. Bay, c'est que la première mêle la langue familière et un parler plus marqué, proche de celui de la paysannerie illettrée, alors que le second utilise la langue familière une seule fois pour ensuite se conformer aux normes du «bon usage» standard.

\section{Le VNA sur le plan fonctionnel}

Malgré ses intentions supposées, Twain a été incapable de calquer le réel linguistique dans sa totalité. De même, ses traducteurs n'ont pas pu recréer un langage marquant spécifiquement la voix noire de son cuvre. Cependant il ne faut pas négliger l'importance, sur le plan implicite, de la présence du VNA. En effet, si

[...] l'allure stéréotypée [d'un sociolecte] résulte d'un transfert direct d'éléments syntactico-sémantiques du code parlé, ceux-ci subissent une systématisation textuelle qui en opère une clôture au deuxième degré - littéraire cette fois - cautionnée par l'émergence de réseaux redondants, d'abord arbitraires (tel aspect de la langue parlée est reproduit et non tel autre), mais qui fonctionnent sur le plan intratextuel en tant que signes motivés, c'est-à-dire comme effet de mimésis sociolectale et/ou idiolectale à fonction identificatrice et idéologique (Lane-Mercier, 1989, p. 172),

à laquelle nous ajouterons, humoristique.

\subsection{Fonction humoristique du VNA}

La fonction humoristique qu'assume le VNA dans The Adventures of Huckleberry Finn, mise en place par la tradition du Sud-ouest ${ }^{14}$, était celle qui sous-tendait toute inscription de vernaculaires régionaux au sein du romanesque. Twain a commencé son roman en ayant à l'idée de conter une histoire pleine d'humour, les

14. «Southwest Humor, beginning in the 1830 s, employed devices for graphically imitating nonstandard speech sounds largely for comic and satiric effect) (Ross, 1989, p. 105). 
personnages y étant innocents, bien que futés, et leur sociolecte, en particulier le VNA, y créant un effet de comique. Il faut toutefois noter que, les personnages acquérant de plus en plus de profondeur à mesure que progresse l'histoire, cet effet de comique ne traverse pas toute l'œuvre. Comme le constate un critique littéraire de l'œuvre de Twain, Henry Nash Smith, «[what] had begun as a comic story developed incipiently tragic implications contradicting the premises of comedy" (1962, p. 114). Un passage émouvant confirme cette remarque. Jim fait part à Huck de la tristesse qu'il a éprouvée après avoir frappé sa petite fille croyant qu'elle lui était désobéissante, alors que la scarlatine l'avait rendue sourde: «Oh, Huck, I bust out a-cryin' en grab her up in my arms, en say, "Oh, de po' little thing! de Lord God Amighty fogive po' ole Jim, kaze he never gwyne to fogive hisself as long's he live!" Oh, she was plumb deef en dumb, Huck, plumb deef en dumb - en I'd ben a'treat'n her so!» $(H F, 126)$. Cependant, l'effet de comique que produit le VNA est tout de même présent au début du roman. Il suffit pour s'en convaincre de jeter un coup d'œil aux premières répliques de Jim et à la situation dans laquelle il se trouve. C'est la nuit, Huck Finn et Tom Sawyer sont sortis sans permission, mais ils font du bruit et Jim les a entendus. Il sort, sachant bien que ce doit être eux, et leur dit: «Who dah?» $(H F, 10)$. Déjà ces paroles nous font sourire. Twain a reproduit, avec ces deux mots seulement, la tendance esthétique propre à son époque qui consistait en la représentation de parlers vernaculaires dans le but de faire rire. Mais les traducteurs ont-ils vu l'importance de cette première réplique qui provoque le rire en raison des deux déviations qu'elle contient, soit phonétique («there» devient «dah») et morphologique (le verbe est absent)? Nétillard l'a rendue par «Qui c'est qui est là?» (Nétillard, p. 12), où - bien qu'il y ait quand même un effort pour donner un effet de comique infantile peut-être -, seule une déviation syntaxique commune est présente («qui c'est» au lieu de la forme correcte «qui est-ce»); et Bay a traduit «Who dah?» par la question - standard à tous points de vue - «Qui est là?» (Bay, p. 188). Aussi, dès la première prise de parole de Jim, l'effet de comique créé en anglais par l'utilisation du VNA est-il perdu dans les deux cas puisque la traduction de Nétillard n'arrive pas à réactualiser, en 
français, un langage proche du VNA, tandis que celle de Bay le gomme complètement.

\subsection{Fonction identificatrice}

G. Lane-Mercier fait ressortir la fonction identificatrice qu'assument les marqueurs sociolectaux au sein du romanesque, identificatrice de la provenance régionale ou du statut social du locuteur: «[...] on conçoit en effet aisément que la représentation des facteurs de prononciation s'avère un lieu privilégié où se cantonnent force opérateurs de mimésis dont la fonction consiste à indiquer de façon emblématique le statut même du sujet d'énoncation [...]» $(1989, \mathrm{p}$. 165). Or, les toutes premières paroles de Jim, qui sont "Who dah?», marquent, à elles seules, son appartenance sociale. Même si le narrateur Huck ne nous avait pas présenté Jim comme étant «Miss Watson's big nigger», l'absence de verbe, la nouvelle graphie du morphème «there», auraient suffi pour indiquer la parole noire, puisque, comme le notait Barthes (1988): «[...] chaque homme est prisonnier de son langage: hors de sa classe, le premier mot le signale, le situe entièrement et l'affiche avec toute son histoire» (p. 59). Lorsque Jim continue, un peu après, il n'y a plus de doutes dans notre esprit, nous savons que Jim est un esclave et qu'il est illettré: "Say - who is you? Whar is you? Dog my cats ef I didn" hear sumf'n. Well, I knows what I's gwyne to do. I's gwyne to set down here and listen tell I hears it agin» $(H F, 10)$. McKay (1982) écrit justement au sujet du VNA qu'il est «[regional] and social [...] and accounts for the differences between Huck and Jim's speech» (p. 163; nos italiques). Sewell (1987, p. 136) souligne aussi le fait que les parlers de Huck, de Tom Sawyer et de Jim étaient motivés par le milieu social de chaque personnage.

Le parler de Jim le caractérise donc comme un esclave qui maîtrise mal le code linguistique. Or, nous l'avons vu, Nétillard a tout de même tenté de rendre les paroles de Jim de manière à ce que nous voyions qu'il connaît mal la langue, mais en ayant recours à un vernaculaire qu'elle a jugé correspondant, c'est-à-dire le parler paysan. Tel a d'ailleurs été le parti-pris de Mimi Perrin, traductrice du roman d'Alice Walker, The Color Purple - pour qui l'accent et 
les expressions rurales peuvent très bien rendre le VNA - et dont Bernard Vidal a étudié et critiqué les implications idéologiques dans son travail sur Zora Neale Hurston et Alice Walker.

La réalité sociale à laquelle renvoie le VNA, celle d'un peuple victime d'esclavage n'est pas celle que vivaient les paysans. Cette pratique rejoint ce que Berman a appelé «l'exotisation des réseaux langagiers vernaculaires» (1985, p. 78), c'est-à-dire le contraire de leur destruction. Selon lui, «[un] vernaculaire ne peut être traduit dans un autre vernaculaire. [...] Une telle exotisation, qui rend l'étranger du dehors par celui du dedans, n'aboutit qu'à ridiculiser l'original» (p. 79). Dans le cas de la traduction de Nétillard, nous n'irions pas jusqu'à affirmer qu'elle ridiculise l'original, mais, par un procédé d'《annexion» (Meschonnic, 1973, p. 308), qu'elle le prive de véhiculer une réalité absolument unique. Aussi le personnage de Jim se trouve-t-il caractérisé comme appartenant au groupe social des paysans de par les tournures et expressions linguistiques propres à ce groupe, mais également à une invention romanesque du personnage noir puisque Nétillard transforme la graphie du substantif «monsieur» en «missié», graphie qui renvoie à la période colonialiste et qui relève d'un parler «petitnègre ${ }^{15}$ " aux connotations bien différentes de celles du VNA. Dès lors, l'alternance des deux registres linguistiques utilisés par Jim, le vernaculaire rural et le parler petit-nègre, produit un texte à la fois moins cohérent et moins homogène que l'original et rejoint par là ce que Berman a appelé «la destruction des systématismes» (1985, pp. 77-78).

15. Comme le fait remarquer Léon-François Hoffmann (1973), qui a consacré une étude intéressante à la représentation du personnage noir dans la littérature française des $\mathrm{XVIII}^{e}$ et XIX ${ }^{e}$ siècles, et dont le point de vue politique est non raciste, «[faire] parler aux Noirs une langue rudimentaire, c'est les traiter en grands enfants, c'est rendre leurs aspirations dérisoires [...]» (p. 107). Précisons cependant que l'occurrence du mot «missié» est la seule marque de ce parler petit-nègre. 
Pour sa part, le parler de Jim dans la traduction de Bay ne semble pas renvoyer au même statut social que l'anglais, puisque Jim vouvoie les autres. Or, selon une étude de Roger Brown et Marguerite Ford (1964, pp. 234-244) qui se sont intéressés aux formules de désignation en anglais américain, un parallèle peut être établi entre la désignation anglaise utilisant un titre avec le nom de famille («title with last name» ou «TLN») et le «vous» français, et entre la désignation anglaise plus familière utilisant le prénom seulement («first name» ou «FN») et le «tu» français. Dans l'original, Jim s'adresse à Huck Finn en l'appelant Huck - ils entretiennent d'ailleurs une relation d'amitié -, mais appelle Tom Sawyer, «Misto Tom» pour «Mister Tom», «Mars Tom» pour «Master Tom ${ }^{16}$ » ou «sah» pour «sir». Nous pourrions donc voir en français Jim tutoyer Huck Finn, mais vouvoyer Tom Sawyer. Or, Bay a choisi l'option du vouvoiement systématique, sauf à deux reprises, sur lesquelles nous reviendrons. Ce vouvoiement nous paraît plus ou moins adéquat, d'une part en raison de la relation amicale qui unit les deux personnages et d'autre part en raison des connaissances linguistiques qu'elle présuppose. En effet, comme le soulignait Bernard Vidal (1991):

L'apprentissage de la langue chez les esclaves ou les indigènes s'est accompli avant tout oralement par mimétisme ou «psittacisme» [...]. Le Noir ne pouvait que reproduire ce qu'il entendait or, chaque fois que son maître lui parlait, il devenait un «tu». Aussi, dans les pays qui furent à une époque colonies françaises, le tutoiement est-il fréquent de la part des habitants; le vouvoiement y est plus rare, et le soin de personnes instruites maîtrisant parfaitement la conjugaison des verbes français. (p. 169, n. 36)

Selon cette remarque, Jim ne peut vouvoyer Huck, pas plus qu'il n'est logique qu'il sache conjuguer les verbes au présent ou au passé

16. Notons que «Misto» et «Mars» sont tous deux traduits par le titre de «monsieur» dans les deux traductions, aucun des traducteurs n'ayant vu ou choisi le titre de "maître» qui, selon nous, correspond bel et bien à «Mars». 
du subjonctif. Toutefois, il est des cas où l'utilisation de désignations différentes est justifiée. Une illustration en est fournie par la retraduction transparente de The Hamlet de William Faulkner par le GRETI. Annick Chapdelaine exprime ainsi le sens du travail des membres du groupe: «Dans notre traduction des conflits des groupes dans le Hameau, nous avons pu reconstruire les rapports de force à l'aide de cet opérateur de mutualité et de non-réciprocité» que représente le choix conscient de «l'alternance du 'tu' et du 'vous'» (à paraître). Nétillard semble avoir aussi choisi de recréer les rapports de place par le biais de ce procédé. Ainsi, elle a respecté la présence ou l'absence de titres, tels Misto, Mars ou sah, et, selon le cas, a choisi le vouvoiement ou le tutoiement. De cette manière, elle a tenu compte des rapports de place tels que Twain les avait établis dans l'original. Dès lors, Jim tutoiera Huck, mais vouvoiera Tom. Nous croyons cependant, comme le préconisait Vidal, que le vouvoiement aurait pu être évité en raison de la caractérisation de Jim dans l'original. En effet, il est évident que Jim est un esclave et un illettré; de plus, quand il s'adresse à des supérieurs, il n'emploie pas toujours le TLN, mais une forme rare, que l'étude de Ford et Brown ne mentionne pas, à savoir le «titre avec le prénom» que constitue la désignation «Misto Tom», par exemple. À elle seule, cette désignation nous semble caractériser toute la voix noire de l'œuvre; elle nous semble participer de sa parlance (Berman, 1985, p. 74). L'étrangeté qu'aurait représentée une réplique du type «dis pas ça, m'sieur Tom» au lieu de «dites pas ça, missié Tom» (Nétillard, p. 341) aurait montré à la fois l'importance des rapports de place et les usages linguistiques propres au personnage de l'esclave, traits qui, d'ailleurs, ne sont pas toujours respectés puisqu'il arrive une fois à Jim d'utiliser le passé simple ${ }^{17}$.

17. Il s'agit d'un passage dans lequel le discours de Jim rapporté par le narrateur a été traduit en discours direct: " - C'est la mort, disait-il. Un jour, que mon père était très malade, quelqu'un de la famille avait pris un oiseau... et mon père mourut; ma vieille grand-mère l'avait bien dit» (Nétillard, p. 67). C'est peut-être le transfert du discours indirect en discours direct qui a poussé Nétillard à avoir recours au passé simple. Une étude intéressante serait à faire, nous semble-t-il, sur cet aspect de la traduction. Y 
Il existe un autre aspect qui peut influer sur les paroles échangées entre des locuteurs et qui concerne le statut social, soit les "contraintes de l'univers de discours ${ }^{18}$ ). Ces contraintes peuvent transformer les répliques des locuteurs selon qu'ils se trouvent en présence de locuteurs qui ne parlent pas le même sociolecte qu'eux, ou selon leur rôle au sein de l'œuvre littéraire. Dans The Adventures of Huckleberry Finn, l'univers de discours exerce des contraintes sur des personnages comme Mary Jane Wilks qui, comme l'a constaté Sewell (1987), «[when] in chapter 28 she assumes the moral stature of Twain's beloved Joan of Arc, [...] undergoes an instantaneous linguistic transformation» (p. 89). Mais ce genre de transformation n'aura pas lieu chez Jim puisque, selon Sewell, il appartient à ce groupe de locuteurs qu'il classe dans le «folk speech», lequel est, chez Twain, «[...] characteristically innocent, averse to the role playing that goes along with the shifts in style adopted easily by the Duke or Tom Sawyery (p. 104). De plus, utilisant un parler qui le caractérise comme un illettré, Jim ne peut pas transformer son discours même s'il est en présence de locuteurs détenteurs d'une langue dite «légitime», ce qui, d'après son statut d'esclave, arrive très rarement. Bourdieu (1982) constate que «[les] locuteurs dépourvus de la compétence légitime se trouvent exclus en fait des univers sociaux où elle est exigée, ou condamnés au silence» ( $p$. 42). C'est pour cette raison, selon nous, que le parler de Jim ne pourra être altéré par les contraintes de l'univers de discours, parce qu'il ne possède ni les compétences sociales, ni les compétences linguistiques nécessaires.

aurait-il des stratégies de la part des traducteurs pour transformer $\mathrm{du}$ discours indirect en discours direct?

18. Nous empruntons cette notion à Lane-Mercier (1989, p. 164), qui, tel qu'elle le rapportait au début de son ouvrage, avait d'abord repris la notion d' (univers de discours» à Catherine KerbratOrecchioni (1980) qui la définit selon deux critères: «les conditions concrètes de la communication» et «les caractères thématiques et rhétoriques du discours, c'est-à-dire en gros les contraintes de 'genre'» (p. 17). 
Or, il semble que Jim ait cette capacité de transformer son sociolecte, dans la traduction de Bay. Jim vouvoie Huck pendant presque tout le roman, sauf deux fois. Une première fois au milieu du livre, Jim croit qu'il est arrivé à Cairo, la ville où il sera libre, et dans sa joie il tutoie Huck. C'est la même situation qui se produit à la fin du roman au moment où Jim est prétendument affranchi. Jim tutoie Huck parce qu'il se sent désormais son égal. Par ses choix de traduction, Bay ne tient pas compte de la caractérisation de Jim dans l'original, où son discours est homogène dans le rapport de familiarité qu'il entretient avec Huck. Comme celle de Nétillard, la traduction de Bay mettrait au jour la tendance déformante qui détruit les systématismes propres à l'œuvre originale en la rendant moins homogène. Or, le tutoiement occasionnel de Jim nous paraît participer d'une profonde incohérence qui altère la logique du texte anglais.

\subsection{Fonction idéologique}

Le VNA tel que Twain se l'approprie dans The Adventures of Huckleberry Finn assume une fonction idéologique qui dépasse le mimétisme, en ironisant le discours hégémonique raciste de sa société. Twain prend une position idéologique critique qui, sur le plan explicite, est constamment diluée dans une série d'épisodes et de paroles actorielles se chargeant d'endosser le discours hégémonique que Twain désapprouve. Prenons, par exemple, ce commentaire de Jim dans lequel il assimile les catégories que la classe dominante utilise pour le décrire (Sewell, 1987, p. 111): «[...] I's rich now, come to look at it. I owns myself, en I's wuth eight hund'd dollars» $(H F, 42)$. Huck endosse également ce préjugé selon lequel les Blancs savent mieux comment agir que les Noirs lorsque, rappelant les idées saugrenues de Tom Sawyer pour libérer Jim (que tous trouvent inutiles et risquées, Huck et Jim inclus), il rapporte l'opinion de Jim ainsi: «Jim couldn't see no sense in the most of it, but he allowed we was white folks and knowed better than him; so he was satisfied and said he would do it all as Tom said» $(H F, 196)$. Le même discours raciste est véhiculé par Aunt Sally qui demande à Huck si un accident maritime a fait des blessés: «'Good gracious! anybody hurt?' No'm. Killed a niger. 'Well, it's lucky; because 
sometimes people do get hurt [...]'» $(H F, 175)$. Ces trois extraits semblent racistes sur le plan explicite, mais ils dénoncent d'une manière tout à fait ironique et implicite le discours du Sud présécessionniste. D'ailleurs l'ironie de Twain est partout présente dans ce texte. En effet, c'est à travers un abject personnage, à la fois ivrogne et batteur d'enfants, Pap Finn, le père de Huck, que Twain fait circuler un ensemble de lieux communs sur les Noirs. Pourtant, sur le plan implicite, ce personnage canalise à lui seul tout le sarcasme de Twain à l'endroit des Blancs et de leur manière de penser. C'est d'ailleurs ce qu'affirme un historien marxiste, Philip S. Foner (1958), pour qui The Adventures of Huckleberry Finn «[...] is filled with devastating thrusts at the whole idea of white supremacy» (p. 208). Or, non seulement Twain dénonce l'esclavage, et un de ses fondements, le racisme, mais son œuvre critique aussi la période post-esclavagiste, celle de la Reconstruction (Kaspi, 1986, p. 199) ${ }^{19}$. Cette période voit l'affranchissement des Noirs, mais leur vie n'en est pas pour autant améliorée.

En 1876 [...] les Noirs du Sud perdent leur droit de vote. La ségrégation dans les écoles, les hôpitaux, les moyens de transport devient peu à peu une habitude, jusqu'au moment où, en 1896, la Cour suprême en reconnaît la légalité. [...] L'esclavage a disparu; la discrimination lui succède. (Kaspi, 1986, p. 209)

Il semble que The Adventures of Huckleberry Finn reproduise cette aberration sociale selon laquelle les Noirs ont une liberté que nous pourrions qualifier d'abstraite ou d'artificielle, puisqu'elle ne leur donne aucun droit concret. Lorsque Tom Sawyer prend en charge, d'une manière tout à fait extravagante, l'évasion de Jim, il ignore deux réalités importantes concernant Jim: la première est qu'il sait que Miss Watson l'a affranchi, mais il ne le lui dit pas pour avoir le plaisir de le libérer; la seconde est qu'il n'écoute pas les doléances de Jim sur les mauvais traitements qu'il lui fait subir. Ce

19. On appelle «Reconstruction», la période trouble qui a suivi la guerre de Sécession (1860-1865), période durant laquelle on tente de rebâtir les bases politiques, économiques et sociales d'un pays divisé depuis plus de cinq ans. 
sont là des preuves, à notre avis, que la voix de Jim, même si elle est présente formellement, n'est pas prise en considération. Twain démontre, par cette fin douteuse (aucun critique n'a cru à l'affranchissement de Jim par Miss Watson), que la fin de l'esclavage n'égale pas nécessairement liberté et égalité pour les Noirs:

The white man, Tom Sawyer, knows [Jim] is free. But is the Negro ready for freedom? No, says Tom, he is illiterate and must be taught to write, under difficulties, to be sure - with a spoon on a grindstone, or with his own blood. The Negro [...] does not "understand» what must be done to be regular and right in attaining freedom. He must be taught by the superior white man how to become free... (Beaver, 1987, p. 45)

Pour nous, l'utilisation du VNA est une autre preuve de la volonté de Twain de dénoncer la réalité que vivaient ces Noirs affranchis. Dans The Adventures of Huckleberry Finn, le Noir a une voix, sa voix, le VNA, mais il n'est pas entendu; la réalité de la Reconstruction n'était pas autre: le Noir était libre, mais il n'avait pas sa place dans la société américaine. Dès lors, la non-traduction du VNA, ou sa traduction approximative, escamote la fonction idéologique qu'assume le VNA, celle de dénoncer de manière ironique deux situations bien réelles, l'esclavage et la ségrégation qui lui a succédé.

Si nous parlons de non-traduction, c'est que nous avons constaté que Bay s'est parfois permis la liberté de ne pas traduire des passages entiers des répliques de Jim. Passages qui, dans la plupart des cas, démontraient l'intelligence de Jim, sa capacité à élaborer un plan, son sens stratégique (voir Bay, pp. 217-220). Ce gommage de la parole noire nous semble une atteinte véritable à la signifiance de cette œuvre. Autre écart de traduction, Bay s'éloigne souvent du texte anglais. Ainsi, à l'occasion d'une réplique de l'esclave Nat, chargé de la surveillance de Jim qu'on a repris, et qu'il désigne comme étant: «dish-yer runaway nigger» $(H F, 186)$, 
Bay a traduit les mots de Nat par: «ce foutu nègre» (Bay, $\mathrm{p}$. $349)^{20}$. Dans la traduction, l'esclave est vu comme une mauvaise personne, alors qu'en anglais aucun jugement de valeur ne le déprécie: il est seulement en fuite. La fonction idéologique qu'assumait le VNA en anglais est donc déphasée dans la traduction. Par ailleurs, nous avons constaté que ce portrait dépréciatif du Noir était endossé par les paroles du personnage le plus raciste du roman, et que nous avons déjà décrit, Pap Finn. Il s'agit de son grand discours dans lequel il s'en prend au gouvernement qui laisse des Noirs séjourner librement jusqu'à six mois dans des États esclavagistes avant d'être pris par les autorités et considérés comme des esclaves. Ses dernières paroles sont en effet pétries de racisme: «Here's a govment that calls itself a govment, and lets on to be a govment, and thinks it is a govment, and yet's got to set stock-still for six whole months before it can take ahold of a prowling, thieving, infernal, white-shirted free nigger, and -» $(H F, 27)$. Voilà comment Bay a rendu ce pasage: «Ça s'appelle un gouvernement, ça se dit un gouvernement, ça joue au gouvernement, mais leur faut six mois avant de mettre la main au collet d'un sale voleur, rôdeur, d'un vieux singe noir en chemise blanche» (Bay, p. 206). Il est intéressant de noter que Bay n'a pas traduit «free» et que «nigger» est devenu à lui seul «vieux singe noir». Le traducteur nous semble ici dépasser la pensée de Twain. Il n'y avait pas en anglais de «black old monkey». Cette association du Noir au singe charrie à elle seule toute une idéologie raciste qui date surtout, en fait, des siècles précédents ${ }^{21}$. Le traducteur s'est laissé emporter par le discours de Pap Finn. Comme le fait remarquer Marc Angenot (1989), «[il] suffit souvent de s'abandonner à une phraséologie pour

20. Voici la traduction de Nétillard (p. 310): «Ce nèg-là, missié!». Notons que l'anglais ne signalait aucun «sir» ni «mister» dans cette réplique, mais que Nat aura recours à la désignation «sah» à trois reprises à l'intérieur d'une seule prise de parole quelques lignes plus bas.

21. «L'identification burlesque du Nègre au singe deviendra au XIX siècle un des leitmotive du racisme littéraire», Hoffmann (1973, p. 32). 
se laisser absorber par l'idéologie qui lui est immanente» (p. 18). S'éloignant maintes fois de l'original, il semblerait que le traducteur Bay n'ait pu endosser le même projet idéologique que Twain ou qu'il se soit pris au piège de la lecture au premier degré, celle du message explicite pro-esclavagiste pourtant constamment manipulé, subverti et ironisé par toutes sortes de manœuvres, dont la plus évidente est, à notre avis, la présence sur le plan strictement formel, et d'un personnage central noir ${ }^{22}$, et d'une voix qui lui est propre.

En ce qui a trait à la traduction de Nétillard, il semblerait que cette dernière ait endossé sensiblement la même position idéologique que Twain, bien qu'elle ait parfois cru bon d'atténuer l'ironie d'un ou deux passages. Ainsi, elle traduit presque toujours le mot «nigger» par «nègre», mais le remplace parfois par un pronom personnel si le passage en contient une grande quantité. De plus, les derniers mots du discours de Pap Finn sont moins choquants à notre avis que ne l'étaient ceux de l'original puisque la traductrice les a rendus par: «[...] mettre la main sur un voleur de nègre en chemise blanche qui vient rôder par ici...» (Nétillard, p. 44). Or, le syntagme "voleur de nègre» n'est pas aussi clair que l'original et les mots «infernal» et «free»n'ont pas été traduits. Enfin, il n'est pas étonnant de voir chez Nétillard la fameuse phrase de Tom Sawyer qui nous apprend que Jim est «as free as any cretur that walks this earth!» $(H F, 226)$ rendue par «il est aussi libre que toi et moi» (Nétillard, p. 375). Les choix de traduction de Nétillard produisent donc un déphasage par rapport à l'original. Son utilisation du mot «missié» conjuguée avec celle d'un vernaculaire rural, de même que l'adoucissement de certains passages, contribuent à un effet de décentrement ${ }^{23}$ senti à la lecture du texte

22. Foner (1958) considère aussi que «Jim is the real hero of the novel. He is a warm human being, lovable and admirable. His nobility shines through the entire book» (p. 205).

23. Nous ne donnons pas ici à «Décentrement» le sens mélioratif que lui attribue Meschonnic, (1973, p. 308), mais plutôt celui de distance, d'éloignement par rapport à l'original. 
français et donnent à lire, comme c'est le cas pour les choix de traduction de Bay d'ailleurs, un implicite autre.

\section{Conclusion}

Deux hypothèses principales expliqueraient, selon nous, les lacunes que contiennent les deux traductions que nous avons analysées. La première, extéro-déterminée, serait liée au contexte socio-historique dans lequel ont été produites les traductions et aux exigences des maisons d'édition. Par contexte socio-historique, nous entendons les exigences esthétiques et littéraires de l'époque qui ont pu influer sur les choix faits par les traducteurs qui n'étaient peut-être pas habitués à voir des sociolectes représentés dans une œuvre romanesque. En effet, comme le souligne Lane-Mercier (1990):

Historiquement marquées vis-à-vis d'une forme perçue comme normative, ces inscriptions [sociolectales] se posent en tant qu'infraction et, comme telles, portent atteinte tantôt à l'institution littéraire (qui a toujours prôné la standardisation de l'orthographe ainsi que tout un code de «bienséances verbales» - en témoigne, jusqu'au mouvement naturaliste, l'exclusion des parlers populaires et argotiques du domaine littéraire), tantôt au scriptural, senti comme supérieur au code oral parce que plus homogène [...]. ( $\mathrm{p}$. 45)

De plus, les éditeurs ont fort probablement eu leur mot à dire en ce qui a trait aux traductions, sur le double plan du fond et de la forme. Une maison d'édition qui, comme c'est le cas pour la traduction de Nétillard, publie de la littérature pour la jeunesse, peut choisir ${ }^{24}$ de publier un texte dont le contenu ne choquera pas ses lecteurs en demandant l'élimination d'expressions jugées vulgaires ou racistes. Mais encore, la maison d'édition peut exiger la suppression de longs passages jugés inintéressants pour des adolescents. Cette dernière option a d'ailleurs été choisie par la collection «Folio junior» pour

24. Rappelons que la première parution de la traduction de Nétillard avait été publiée aux Éditions Hier et Aujourd'hui, maison d'édition qui ne visait pas nécessairement un public d'adolescents. 
la traduction française de Uncle Tom's Cabin ${ }^{25}$. Dans le cas de Bay, il serait possible que les éditeurs aient demandé un texte plus court et qui répondait plus spécifiquement aux exigences linguistiques de leur maison d'édition.

La seconde hypothèse, intéro-déterminée celle-là, serait liée aux stratégies auxquelles peuvent avoir recours les traducteurs. En fait, ces derniers pourraient, d'une part, reproduire l'original à l'aide d'《effets de réel» (Barthes, 1984, p. 186) comme la recréation (il ne s'agit pas ici d'imitation pure et simple) des systématismes de l'œuvre, de ses rythmes, de ses réseaux signifiants sous-jacents, de ses réseaux langagiers vernaculaires et de ses locutions; et, d'autre part, transformer, altérer l'original en ne tenant pas compte des systématismes, des rythmes, etc., de l'œuvre. D'après nos interprétations, autant Bay que Nétillard ont choisi la seconde option, créant (dans le cas de Nétillard surtout) un nouveau langage pour Jim, effaçant (dans le cas de Bay seulement) des parties de son discours, privant l'œuvre de son projet idéologique profond: donner une voix, une identité donc, à des êtres qui, jusque-là, en avaient été privés.

\section{Références}

ANGENOT, Marc (1989). 1889. Un état du discours social, Longueuil, Le Préambule.

BARTHES, Roland (1984). Le Bruissement de la langue. Essais critiques IV, Paris, Seuil.

25. Notons au passage que sur un total de 45 chapitres dans l'œuvre originale de Harriet Beecher Stowe (1850), Uncle Tom's Cabin (New York, Random House, 1938, 552 p.), il ne reste, dans la traduction française, que 37 chapitres. Dans cette version (Gallimard, «Folio-junior», 1982, 395 p.), le nom du traducteur ou de la traductrice n'est pas indiqué. 
(1988). Le Degré zéro de l'écriture, suivi de Nouveaux essais critiques, Paris, Seuil. $1^{\text {ires }}$ éd.: 1953 et 1972 (respectivement).

BEAVER, Harold (1987). Huckleberry Finn, London, Unwin Hyman.

BERMAN, Antoine (1985). «La Traduction et la lettre ou l'auberge du lointain», les Tours de babel, Mauvezin, Trans-Europ-Repress.

(1984). L'Épreuve de l'étranger. Culture et traduction dans l'Allemagne romantique. Paris, Gallimard.

BLAIR, Walter (1960). Mark Twain \& 'Huck Finn', Berkeley, California, University of California Press.

BOURDIEU, Pierre (1982). Ce que parler veut dire, Paris, Fayard.

BRADLEY, Sculley, et al. (1977). Samuel Langhorne Clemens, Adventures of Huckleberry Finn. An Authoritative Text Backgrounds and Sources Criticism, edited by Sculley Bradley, et al. New YorkLondon, W.W. Norton \& Company, Second Edition.

BRISSET, Annie (1990). Sociocritique de la traduction: théâtre et altérité au Québec, 1968-1988, Longueuil, Le Préambule.

BROWN, Roger W., et Marguerite FORD (1964). "Address in American English», Language in Culture and Society, Dell Hymes, dir. New York, Harper and Row.

CHAPDELAINE, Annick (à paraitre). «Reconstructions identitaires en traduction: le conflit des groupes et des langages dans The Hamlet de Faulkner», Palimpsestes.

DEVOTO, Bernard (1942). Mark Twain at Work, Cambridge, Massachusetts, Harvard University Press.

FONER, Philip S. (1958). Mark Twain: Social Critic, New York, International Publishers. 
HOFFMANN, Léon-François (1973). Le Nègre romantique, Paris, Payot.

KASPI, André (1986). Les Américains. I. Naissance et essor des États-Unis. 1607-1945, Paris, Seuil.

KERBRAT-ORECCHIONI, Catherine (1980). L'Énonciation: de la subjectivité dans le langage, Paris, Armand Colin.

LABOV, William (1972). Sociolinguistic Patterns, Philadelphia, University of Pennsylvania Press; Sociolinguistique, traduit par Alain Kihm, Paris, Minuit, 1976.

LANE-MERCIER, Gillian (1989). La Parole romanesque, OttawaParis, Presses de l'Université d'Ottawa-Klincksieck.

LANE-MERCIER, Gillian (1990). «Pour une analyse du dialogue romanesque», Poétique, 81 (février).

MCKAY, Janet H. (1982). Narration and Discourse in American Realistic Fiction, University of Pennsylvania Press.

MESCHONNIC, Henri (1973). Pour la poétique II, Paris, Gallimard.

PEDERSON, Lee A. (1965-1966). «Negro Speech in The Adventures of Huckleberry Finn'), The Mark Twain Journal, XIII(1), (hiver).

PERRIN, Mimi (1986). «Improviser comme les jazzmen», Actes des deuxièmes assises de la traduction littéraire (Arles, 1985), Atlas, Actes Sud.

ROSS, Stephen M. (1989). Fiction's Inexhaustible Voice. Speech and Writing in Faulkner, Athens and London, The University of Georgia Press. 
SEWELL, David R. (1987). Mark Twain's Languages. Discourse, Dialogue, and Linguistic Variety, Berkeley-Los Angeles-London, University of California Press.

SMITH, Henry Nash (1962). Mark Twain. The Development of a Writer. Cambridge, Massachusetts, The Belknap Press of Harvard University Press.

SMITH, Henry Nash, et al., dir. (1960). The Mark Twain-Howells Letters, Vol. 1. Cambridge, Massachusetts, Harvard University Press.

VIDAL, Bernard (1991). «Plurilinguisme et traduction - Le vernaculaire noir américain: enjeux, réalité, réception à propos de The Sound and the Fury», TTR, IV(2), pp. 151-188.

WHITFIELD, Agnès, et Gregory LESSARD (1991). «Le 'Romanjoual': Tremblay et Beaulieu», Literary Genres / Les genres littéraires, I.S. MacLaren et C. Potvin, dir. Edmonton, Research Institute for Comparative Literature, University of Alberta.

\section{Annexe \\ Texte anglais}

Jim raconte à Huck les raisons pour lesquelles il a pris la fuite:

«Well, you see, it'uz dis way. Ole Missus - dat's Miss Watson - she pecks on me all the time, en treats me pooty rough, but she awluz said she wouldn' sell me down to Orleans. But I noticed dey wus a nigger trader roun' de place considable, lately, en I begin to git oneasy. Well, one night I creeps to de do', pooty late, en de do' warn't quite shet, en I hear ole missus tell de widder she gwyne to sell me down to Orleans, but she didn' want to, but she could git eight hund'd dollars for me, en it'uz sich a big stack o'money she couldn' resis'. De widder she try to git her to say she wouldn' do it, but I never waited to hear de res'. I lit out mighty quick, I tell you. $(H F, 39)$ 


\section{Version de Nétillard}

- Eh bien! c'est comme ça que c'est arrivé: la patronne, Miss Watson, tu sais, elle était tout le temps à me dire des choses et à me maltraiter et à répéter qu'elle allait me vend' à La Nouvelle-Orléans. Mais voilà que j'avais remarqué un marchand d'esclaves qui venait souvent à la maison, ces derniers temps, et je commençais à avoir peur. Et un soir, tard, quand je revenais sans faire de bruit, $j$ 'ai entendu la patronne derrière la porte qui n'était pas tout à fait fermé; elle disait à la Veuve qu'elle n'était pas trop décidée à me vend' à La Nouvelle-Orléans, mais qu'on lui proposait huit cents dollars pour moi et qu'elle ne pouvait pas résister à tout ce tas d'argent. La Veuve essayait de lui faire changer d'avis, mais j'ai pas entendu la suite. J'ai filé, et au galop. (pp. 64-65)

\section{Version de Bay}

- Voilà comment ça s'est fait. La vieille miss Watson, elle était toujours à me chercher noise. Elle avait bien dit qu'elle me vendrait pas à La Nouvelle-Orléans; mais j'ai remarqué que, depuis quelque temps, il y avait dans la région un marchand d'esclaves qui se démenait beaucoup. Ça m'a fait réfléchir. Là-dessus, un soir, tard, en passant près de la porte de la salle, qu'était pas bien fermée, j'entends la vieille dire à la veuve qu'elle avait l'occasion de me vendre huit cents dollars, et qu'elle était très tentée de le faire. J'ai pas eu besoin d'en entendre plus. Je me suis cavalé, je vous dis que ça. (pp. 217-218)

Notons que les deux traductions françaises contiennent un contresens, celle de Nétillard qui fait dire à Jim que Miss Watson désirait le vendre, alors qu'en anglais elle ne veut pas; et celle de Bay qui fait dire à Jim que Miss Watson était très tentée de le faire, alors qu'elle était très tentée par l'argent, ce qui est différent.

RÉSUMÉ: Problèmes de traduction du vernaculaire noir américain: le cas de The Adventures of Huckleberry Finn - Cet article propose une analyse du rôle dévolu au vernaculaire noir américain (VNA) par Mark Twain dans The Adventures of HuckleberryFinn et du traitement qui en a été fait par deux traducteurs français (Suzanne Nétillard, 1948/1973/1985, et André Bay, 1961/1990). L'auteure démontre que la transcription du VNA 
par Twain répond à deux «tendances esthético-cognitives divergentes» (Lane-Mercier). La première, «philologique», où Twain tente, sans vraiment y parvenir en raison de certains effets de clôture, de rendre compte du parler des personnes de race noire dans l'extratexte; la seconde, «artistique», où il cherche à subvertir, à travers sa représentation du VNA sur le plan scriptural, le discours socio-idéologique propre à sa société. En effet, le VNA assume plusieurs fonctions dans The Adventures of HuckleberryFinn: sur le plan esthétique, il crée, au début du roman, un effet de comique; sur le plan social, il identifie le locuteur à son milieu; et sur le plan idéologique, il exprime la position de l'auteur sur l'esclavage et la ségrégation. Or, la tradition française du bien-écrire étant très présente à l'esprit des traducteurs, ces derniers ont plus ou moins pu recréer graphiquement en français un langage caractérisant la voix noire tel que Twain l'avait fait en anglais. Partant, si le VNA n'est pas représenté formellement, toute l'idéologie sous-jacente à sa présence est du mềme coup atténuée, si ce n'est complètement perdue.

\section{ABSTRACT: Problems in Translating Black English Vernacular: The} Case of The Adventures of Huckleberry Finn - This article aims to analyse both the role ascribed to Black English Vernacular (BEV) by Mark Twain in The Adventures of Huckleberry Finn and its treatment by two French translators (Suzanne Nétillard, 1948/1973/1985, and André Bay, 1961/1990). The author demonstrates that Twain's transcription of BEV corresponds to two "divergent esthetic/cognitive trends" (Lane-Mercier). On one hand, a "philological" one, by which Twain attempts - without actually succeeding, because of certain effects of closure - to account for the speech of Black persons in the extratext, and on the other, an "artistic" one, where he seeks, through his written representation of BEV, to subvert the socioideological discourse of his time. BEV indeed takes on a number of functions in The Adventures of Huckleberry Finn: in esthetic terms, it creates a comic effect at the beginning of the novel; in social terms, it identifies the speaker with his or her milieu; and in ideological terms, it conveys the author's position with regard to slavery and segregation. Since the translators may have had the French tradition of "proper writing" in mind, they have been only roughly successful in graphically recreating, in French, a language that characterizes Black voice as Twain had done in English. Therefore, the author concludes, failing to formally represent BEV in a text causes the entire ideology underlying it to be diluted, if not lost completely. 